\title{
Modulated linear dynamics of nanobeams accounting for higher gradient effects
}

\author{
Dario Abbondanza ${ }^{a}$, Daniele Battista ${ }^{a}$, Francescogiuseppe Morabito $^{a}$, Chiara Pallante ${ }^{a}$, \\ Raffaele Barretta ${ }^{b}$, Raimondo Luciano ${ }^{c}$, Francesco Marotti de Sciarra ${ }^{b}$, Giuseppe Ruta ${ }^{d}$ \\ ${ }^{a}$ Dipartimento di ingegneria meccanica e aeronautica, "La Sapienza", Rome, Italy \\ ${ }^{b}$ Dipartimento di strutture per l'ingegneria e l'architettura, Università degli Studi di Napoli "Federico II", Naples, Italy \\ ${ }^{c}$ Dipartimento di ingegneria civile e meccanica, Università degli Studi di Cassino e del Lazio Meridionale, Cassino (FR), Italy \\ ${ }^{d}$ Dipartimento di ingegneria strutturale e geotecnica, "La Sapienza", Rome, Italy
}

\begin{abstract}
We present some numerical results for the linear dynamics of nanobeams modulated by an axial force, basing on a recent proposal of literature that encompasses both the standard nonlocal elasticity, according to Eringen, and second-order strain elasticity. Three different possibilities for the elastic potential energy provide different responses that highlight the contributions of nonlocality and strain gradient, plus their combination. An axial force affects the linear stationary dynamics of such nanobeams, inducing suitable variation of the natural angular frequencies for benchmark cases, until static buckling occurs when the natural angular frequency vanishes. Effects of the various elastic potentials on this modulation are investigated and thoroughly commented.
\end{abstract}

\section{Introduction}

In recent years the use of nanobeams as sensors or actuators in many fields of physics and applied physics has seen a remarkable increase, see e.g. [1, 2, 3, 4]. Going down to such a small length scale, the usual structural models are not sufficient, even in the usual approximation of 'small' displacements and strain with respect to the length of the considered specimen. Several models attempting to describe the constitutive response of elastic nanobeams have been proposed in the literature, among which we may quote innovative nonlocal strategies $[5,6,7,8,9,10$, 11], gradient theories $[12,13,14,15,16,17]$, couple stress and surface energy approaches [18, 19, 20, 21]. A comprehensive collection of results on nanostructures can be found in recent review articles and books $[22,23,24,25,26]$ and references therein. Recently, three of the authors of the present paper proposed an elastic potential encompassing both the effects of the usual Eringen model of nonlocal elasticity and a reasonable formulation of strain gradient elasticity [27]. This potential manages to see both effects as additional curvatures and secondorder strains in a standard quadratic expression. Some results of this proposal have been proposed to look for the static solutions of benchmark cases [28].

In this paper we rely on a standard purely flexible (a.k.a. Euler-Bernoulli) beam model, and adopt the elastic potential energy densities introduced in [27]. By means of usual variational procedures we derive the field equations for the linear dynamics of nanobeams subjected to an axial force, which is known to act as a modulating factor for the apparent global stiffness of the beam. Indeed, the effects of an axial load on the natural vibration of beams is known since Woinowsky-Krieger [29], and has been investigated also in the more recent contributions by Bishop and Price [30], Bokaian [31, 32], and Stephen [33]. Roughly speaking, an axial load adds a geometric contribution to the material stiffness of the beam, thus affecting its natural frequencies. Tensile forces increase the total stiffness of the beam, 
compressive forces do the opposite, and make the natural frequencies decrease until they vanish and induce static buckling (see, e.g., Bazant and Cedolin [34]). Thus, the axial force works as a control parameter for the natural angular frequencies of the beams, which depend directly on the stiffness of the beam, and which we find by means of a Galerkin approach in some benchmark cases.

In the following, we will resume the essentials of the beam model, we will present the derivation of the field equations for the considered problems, then we will present the results that we obtained for the fundamental natural angular frequency and for its variation with respect to the variation of some meaningful material parameters under some usual boundary conditions. We will thoroughly comment the obtained results in the light of the known results of literature for the usual Eringen model, as well as for strain gradient elasticity, and we will sketch some future developments of this research.

\section{A purely flexible model for nanobeams}

Let us consider a nanobeam that in its reference configuration has the centroidal axis occupying a straight segment of length $l$ on the $x$-axis of a Cartesian coordinate system with the origin at the left end of the beam. We will study a plane behaviour, and assume that the scalar components of the infinitesimal displacements of the beam, seen as a three-dimensional cylinder, are described by

$$
u(x, y, z, t)=-y \frac{d v(x, t)}{d x}, \quad v(x, y, z, t)=v(x, t), \quad w(x, y, z, t)=0
$$

where $t$ is the time. The functions in eq. (1) express the planarity of the motion, plus the conservation of plane cross-sections. The latter rotate by the same amount expressing the slope of the deflected axis of centroids, thus remain orthogonal to the beam axis in every deformed shape. The standard definitions for the infinitesimal three-dimensional strain, i.e.,

$$
\varepsilon_{i j}=\frac{1}{2}\left(\frac{\partial u_{i}(x, y, z, t)}{\partial x_{j}}+\frac{\partial u_{j}(x, y, z, t)}{\partial x_{i}}\right), \quad i, j=1,2,3
$$

provide that all deformation measures vanish but the elongation of the beam along its axis, given by

$$
\varepsilon_{i i}=\varepsilon(x, y, z, t)=\varepsilon(x, y, t)=\frac{\partial u(x, y, z, t)}{\partial x}=-y \frac{d^{2} v(x, t)}{d x^{2}}=-y \chi(x, t)
$$

where $\chi(x, t)$ is the bending curvature of the beam at the point corresponding to the abscissa $x$. For the sake of simplicity of notation, the subscripts were dropped in the definition of the elongation in eq. (3), since no other strain measure is present. In addition, for the same reason the variables on which the indicated fields depend will be dropped from notation when no ambiguity arises.

Basing on the proposal in [27], we suppose that the material response of the nanobeam is given by three different elastic potential energy densities $\mathcal{U}_{i}, i=1,2,3$, which are given by

$$
\left(\begin{array}{l}
\mathcal{U}_{1} \\
\mathcal{U}_{2} \\
\mathcal{U}_{3}
\end{array}\right)=\frac{1}{2} E \varepsilon^{2}\left(\begin{array}{l}
1 \\
1 \\
1
\end{array}\right)+\frac{1}{2} E \frac{d \varepsilon^{2}}{d x}\left(\begin{array}{c}
c^{2} \\
\alpha^{2} c^{2} \\
\alpha^{2} c^{2}
\end{array}\right)+\frac{q}{A} \chi(\varepsilon)\left(\begin{array}{c}
\alpha^{2} c^{2} \\
c^{2} \\
c^{2}
\end{array}\right)+\frac{d q}{d x} \frac{1}{A} \frac{d \chi(\varepsilon)}{d x}\left(\begin{array}{c}
0 \\
0 \\
\alpha^{2} c^{4}
\end{array}\right)
$$

where $E$ is Young's modulus of local elasticity, $c$ is the material parameter accounting for nonlocal elasticity, according to Eringen, $\alpha$ is a participation factor among the three energetic forms, $A$ is the 
area of the cross-section $\mathcal{S}$ of the beam, and $q(x)$ is the static load, directly proportional to the second derivative of the contact bending couple, thus of the axial stress; more details can be found in [27]. It is apparent from eq. (4) that the effect of nonlocal elasticity is taken into account as a kind of non-elastic additional curvature, in the third addend of the potential energy densities $\mathcal{U}_{i}$; moreover, strain gradient elasticity is introduced in the second and fourth addend of the potential energy densities $\mathcal{U}_{i}$, and is reduced to the same form as the first and third addend, simply through a material length that is made to depend on Eringen nonlocal constitutive parameter $c$ by the non-dimensional participation factor $\alpha$. This choice implies the assumption that no strain gradient elasticity effects arise if nonlocal elasticity is not present, thus reducing to ordinary local, first gradient elasticity.

The equations of mechanical balance are obtained by standard calculus of variations, employing Hamilton's principle of least action over a time interval $t \in[0, T]$

$$
\int_{0}^{T}(\delta U-\delta W-\delta K) d t=0
$$

where $\delta U$ is the variation of potential energy, $\delta W$ is the work spent by the external loads, and $\delta K$ is the variation of kinetic energy. The potential energy is simply the integral sum of the potential energy density over the domain $\mathcal{D}=\mathcal{S} \times[0, l]$ occupied by the cylinder constituting the beam, thus, with regard to eq. (4), its variation is given by

$$
\delta U=\int_{\mathcal{D}}\left\{E \varepsilon \delta \varepsilon\left(\begin{array}{l}
1 \\
1 \\
1
\end{array}\right)+E \frac{d \varepsilon}{d x} \delta \frac{d \varepsilon}{d x}\left(\begin{array}{c}
c^{2} \\
\alpha^{2} c^{2} \\
\alpha^{2} c^{2}
\end{array}\right)+\frac{q(x)}{A} \frac{\partial \chi}{\partial \varepsilon} \delta \varepsilon\left(\begin{array}{c}
\alpha^{2} c^{2} \\
c^{2} \\
c^{2}
\end{array}\right)+\frac{d q(x)}{d x} \frac{1}{A} \frac{\partial \frac{d \chi}{d x}}{\partial \frac{d \varepsilon}{d x}} \delta \frac{d \varepsilon}{d x}\left(\begin{array}{c}
0 \\
0 \\
\alpha^{2} c^{4}
\end{array}\right)\right\} d V
$$

The work spent by the external loads consists of two addends, one of which is the contribution provided by transverse distributed load $p(x, t)$ per unit length of the beam, and the other is the contribution of a modulating axial load $N_{0}$. The latter is known to be linear in the square of the slope of the beam axis [35], so that the work spent by the external loads is

$$
\delta W=\int_{0}^{l} p(x, t) \delta v(x, t)\left(\begin{array}{l}
1 \\
1 \\
1
\end{array}\right) d x+\int_{0}^{l} N_{0} \frac{d \delta v(x, t)}{d x}\left(\begin{array}{l}
1 \\
1 \\
1
\end{array}\right) d x
$$

where $N_{0}$ is assumed positive if it acts in compression, and $p(x, t)$ is the sum of a permanent load $q(x)$ and of a load possibly varying with time $q_{e}(x, t)$, that is,

$$
p(x)=q(x)+q_{e}(x, t)=a f(x)+a_{e} f_{e}(x, t)
$$

where the factors $a, a_{e}$, both having dimensions of a force per unit length, act as magnifying multipliers, while the functions $f(x), f_{e}(x, t)$ provide the shape of the external load along the beam axis, with no physical dimensions. This multiplicative decomposition will be useful later for the analysis of benchmark cases by non-dimensional equations, providing results independent of particular values for the involved physical quantities. 
The kinetic energy is assumed to take into account both axial and transverse variations of infinitesimal displacements with time, so that its variation is given by

$$
\begin{aligned}
\delta K & =\int_{\mathcal{D}} \rho\left[\dot{u} \delta \dot{u}\left(\begin{array}{l}
1 \\
1 \\
1
\end{array}\right)+\dot{v} \delta \dot{v}\left(\begin{array}{l}
1 \\
1 \\
1
\end{array}\right)\right] d V=\int_{0}^{l} \int_{\mathcal{S}} \rho\left[y^{2} \frac{d \dot{v}}{d x} \delta \frac{d \dot{v}}{d x}\left(\begin{array}{l}
1 \\
1 \\
1
\end{array}\right)+\dot{v} \delta \dot{v}\left(\begin{array}{l}
1 \\
1 \\
1
\end{array}\right)\right] d A d x= \\
& =\int_{0}^{l} \rho\left[I \frac{d \dot{v}}{d x} \delta \frac{d \dot{v}}{d x}\left(\begin{array}{l}
1 \\
1 \\
1
\end{array}\right)+A \dot{v} \delta \dot{v}\left(\begin{array}{l}
1 \\
1 \\
1
\end{array}\right)\right] d x
\end{aligned}
$$

where a superimposed dot stands for the derivative with respect to time, $\rho$ is the mass density per unit volume of the material of the beam, and $I$ is the second moment of area of the cross-section of the beam with respect to the $z$-axis of the chosen coordinate system. Remark that, owing to this constitutive position for the inertia actions, in eq. (9) both transverse (i.e., relative to the deflection of the beam axis) and rotary (i.e., relative to the rigid rotation of the beam cross-sections) inertia are accounted for, thus presenting a general outlook on the possibilities of natural vibration.

Performing an usual variational procedure, by standard operations, integration by parts, and localisation arguments, one obtains the field equation for the modulated linear dynamics of nanobeams, that is, the mechanical balance equation in terms of the displacement fields, having accounted for the constitutive representations of elastic potential, work of external loads, and kinetic energy

$$
\begin{array}{r}
E I \frac{d^{6} v}{d x^{6}}\left(\begin{array}{c}
c^{2} \\
\alpha^{2} c^{2} \\
\alpha^{2} c^{2}
\end{array}\right)-E I \frac{d^{4} v}{d x^{4}}\left(\begin{array}{l}
1 \\
1 \\
1
\end{array}\right)-\frac{d^{2} q}{d x^{2}}\left(\begin{array}{c}
\alpha^{2} c^{2} \\
c^{2} \\
c^{2}
\end{array}\right)+\frac{d^{4} q}{d x^{4}}\left(\begin{array}{c}
0 \\
0 \\
\alpha^{2} c^{4}
\end{array}\right)+ \\
+\rho I \frac{d^{2} \ddot{v}}{d x^{2}}\left(\begin{array}{l}
1 \\
1 \\
1
\end{array}\right)-\rho A \ddot{v}\left(\begin{array}{l}
1 \\
1 \\
1
\end{array}\right)-N_{0} \frac{d^{2} v}{d x^{2}}\left(\begin{array}{l}
1 \\
1 \\
1
\end{array}\right)+p\left(\begin{array}{l}
1 \\
1 \\
1
\end{array}\right)=0
\end{array}
$$

The field equation (10) is completed by the boundary equations, either of kinematic

$$
\left.\delta v\right|_{0} ^{l}=0,\left.\quad \frac{d(\delta v)}{d x}\right|_{0} ^{l}=0,\left.\quad \frac{d^{2}(\delta v)}{d x^{2}}\right|_{0} ^{l}=0
$$

or mechanic nature at the ends of the beam, i.e., $x=0, x=l$

$$
\begin{aligned}
& E I\left[\frac{d^{5} v}{d x^{5}}\left(\begin{array}{c}
c^{2} \\
\alpha^{2} c^{2} \\
\alpha^{2} c^{2}
\end{array}\right)-\frac{d^{3} v}{d x^{3}}\left(\begin{array}{l}
1 \\
1 \\
1
\end{array}\right)\right]-\frac{d q}{d x}\left(\begin{array}{c}
\alpha^{2} c^{2} \\
c^{2} \\
c^{2}
\end{array}\right)+\frac{d^{3} q}{d x^{3}}\left(\begin{array}{c}
0 \\
0 \\
\alpha^{2} c^{4}
\end{array}\right)+\rho I \frac{d \ddot{v}}{d x}\left(\begin{array}{l}
1 \\
1 \\
1
\end{array}\right)-N_{0} \frac{d v}{d x}\left(\begin{array}{l}
1 \\
1 \\
1
\end{array}\right)=T\left(\begin{array}{l}
1 \\
1 \\
1
\end{array}\right) \\
& E I\left[\frac{d^{4} v}{d x^{4}}\left(\begin{array}{c}
c^{2} \\
\alpha^{2} c^{2} \\
\alpha^{2} c^{2}
\end{array}\right)-\frac{d^{2} v}{d x^{2}}\left(\begin{array}{l}
1 \\
1 \\
1
\end{array}\right)\right]-q\left(\begin{array}{c}
\alpha^{2} c^{2} \\
c^{2} \\
c^{2}
\end{array}\right)+\frac{d^{2} q}{d x^{2}}\left(\begin{array}{c}
0 \\
0 \\
\alpha^{2} c^{4}
\end{array}\right)=-M\left(\begin{array}{l}
1 \\
1 \\
1
\end{array}\right) \\
& E I \frac{d^{3} v}{d x^{3}}\left(\begin{array}{c}
c^{2} \\
\alpha^{2} c^{2} \\
\alpha^{2} c^{2}
\end{array}\right)+\frac{d q}{d x}\left(\begin{array}{c}
0 \\
0 \\
\alpha^{2} c^{4}
\end{array}\right)=0
\end{aligned}
$$

where $T, M$ are the transverse force and bending moment applied at the ends of the beam by contact. 
Remark that the introduction of a constitutive relation accounting for both nonlocal and strain gradient elasticity turned the usual fourth-order ordinary differential equation in space for EulerBernoulli beams, a.k.a. "elastica", into a sixth-order ordinary differential equation in space. Eq. (10) presents only even derivatives with respect to space and time, thus has the same structure of Euler's "elastica". This implies that, in principle, similar strategies may be adopted in the search of a solution in both cases. The external load contributes in the field equation (10) firstly by the whole quantity $p(x, t)$, coming from the work $\delta W$; secondly by the conservative part $q(x)$, which enters the elastic potential energy (4). It is worth noting that when the nonlocal elasticity parameter $c$ and the participation factor $\alpha$ of strain gradient elasticity vanish, the field equation (10) reduces to Euler's "elastica".

Note that the natural boundary conditions enlarge those for the "elastica". On the one hand, there are kinematical conditions for the displacement and the slope of the beam axis, plus a condition on the second spatial derivative of the transverse displacement, deriving from the strain gradient elasticity in the potential energy densities (4). On the other hand, there are mechanical conditions expressing the contact force and couple in terms of the static load $q(x)$ entering the constitutive relations, of the transverse displacement, the modulating axial force, and inertia, plus a condition on the third spatial derivative of the transverse displacement and the first spatial derivative of the static load. Again, it is apparent that when the nonlocal elasticity parameter $c$ and the participation factor $\alpha$ of strain gradient elasticity vanish, the boundary conditions (11), (12) reduce to those of Euler's "elastica".

It is usual to perform parametric investigations by reducing the field equations and the boundary conditions of a given mechanical problem to a non-dimensional form, in order to abstract from particular values of the physical quantities involved and to follow global behaviour depending on suitable non-dimensional ratios. On this purpose, let us introduce the following non-dimensional quantities

$$
\xi=\frac{x}{l}, \quad \hat{v}=\frac{v}{l}, \quad \tau=\frac{c}{l}, \quad \hat{t}=t \sqrt{\frac{E I}{\rho A l^{4}}}, \quad \beta=\frac{a l^{3}}{E I}, \quad \beta_{e}=\frac{a_{e} l^{3}}{E I}, \quad \hat{N}=\frac{N_{0} l^{2}}{E I}, \quad \gamma=\frac{I}{A l^{2}}
$$

The non-dimensional abscissa $\xi$ spans the fixed domain $[0,1]$; the non-dimensional transverse displacement $\hat{v}$ is rescaled with respect to the length of the beam axis; the non-dimensional time is rescaled with respect to a characteristic time interval depending on the material and geometric characteristics of the beam; the non-dimensional load amplitudes $\beta, \beta_{e}$ and modulating force $\hat{N}$ are rescaled with respect to the bending stiffness of the beam in local elasticity; and the slenderness ratio $\gamma$ equals the square of the ratio of the gyration radius of the cross-section to the length of the beam axis.

Keeping into account the positions (13), the non-dimensional form of the field equation (10) is

$$
\begin{aligned}
& \frac{d^{6} \hat{v}}{d \xi^{6}}\left(\begin{array}{c}
\tau^{2} \\
\alpha^{2} \tau^{2} \\
\alpha^{2} \tau^{2}
\end{array}\right)-\frac{d^{4} \hat{v}}{d \xi^{4}}\left(\begin{array}{l}
1 \\
1 \\
1
\end{array}\right)-\beta \frac{d^{2} f(\xi)}{d \xi^{2}}\left(\begin{array}{c}
\alpha^{2} \tau^{2} \\
\tau^{2} \\
\tau^{2}
\end{array}\right)+\beta_{e} \frac{d^{4} f(\xi)}{d \xi^{4}}\left(\begin{array}{c}
0 \\
0 \\
\alpha^{2} \tau^{4}
\end{array}\right)+ \\
& \quad+\gamma \frac{d^{2}\left(\frac{d^{2} \hat{v}}{d \hat{t}^{2}}\right)}{d \xi^{2}}\left(\begin{array}{l}
1 \\
1 \\
1
\end{array}\right)-\frac{d^{2} \hat{v}}{d \hat{t}^{2}}\left(\begin{array}{l}
1 \\
1 \\
1
\end{array}\right)-\hat{N} \frac{d^{2} \hat{v}}{d \xi^{2}}\left(\begin{array}{l}
1 \\
1 \\
1
\end{array}\right)+\left(\beta f(\xi)+\beta_{e} f_{e}(\xi, \hat{t})\right)\left(\begin{array}{l}
1 \\
1 \\
1
\end{array}\right)=0
\end{aligned}
$$

where the load shape functions $f, f_{e}$ of eq. (8) are now expressed in terms of non-dimensional quantities. 
The kinematic boundary conditions (11) read

$$
\left.\delta \hat{v}\right|_{0} ^{l}=0,\left.\quad \frac{d(\delta \hat{v})}{d \xi}\right|_{0} ^{l}=0,\left.\quad \frac{d^{2}(\delta \hat{v})}{d \xi^{2}}\right|_{0} ^{l}=0
$$

The mechanical boundary conditions (12) read

$$
\begin{aligned}
& \frac{d^{5} \hat{v}}{d \xi^{5}}\left(\begin{array}{c}
\tau^{2} \\
\alpha^{2} \tau^{2} \\
\alpha^{2} \tau^{2}
\end{array}\right)-\frac{d^{3} \hat{v}}{d \xi^{3}}\left(\begin{array}{l}
1 \\
1 \\
1
\end{array}\right)-\beta \frac{d f}{d \xi}\left(\begin{array}{c}
\alpha^{2} \tau^{2} \\
\tau^{2} \\
\tau^{2}
\end{array}\right)+\beta \frac{d^{3} f}{d \xi^{3}}\left(\begin{array}{c}
0 \\
0 \\
\alpha^{2} \tau^{4}
\end{array}\right)+\gamma \frac{d\left(\frac{d^{2} \hat{v}}{d \hat{t}^{2}}\right)}{d \xi}\left(\begin{array}{l}
1 \\
1 \\
1
\end{array}\right)-\hat{N} \frac{d \hat{v}}{d \xi}\left(\begin{array}{l}
1 \\
1 \\
1
\end{array}\right)=\hat{T}\left(\begin{array}{l}
1 \\
1 \\
1
\end{array}\right) \\
& \frac{d^{4} \hat{v}}{d \xi^{4}}\left(\begin{array}{c}
\tau^{2} \\
\alpha^{2} \tau^{2} \\
\alpha^{2} \tau^{2}
\end{array}\right)-\frac{d^{2} \hat{v}}{d \xi^{2}}\left(\begin{array}{l}
1 \\
1 \\
1
\end{array}\right)-\beta f\left(\begin{array}{c}
\alpha^{2} \tau^{2} \\
\tau^{2} \\
\tau^{2}
\end{array}\right)+\beta \frac{d^{2} f}{d \xi^{2}}\left(\begin{array}{c}
0 \\
0 \\
\alpha^{2} \tau^{4}
\end{array}\right)=-\hat{M}\left(\begin{array}{l}
1 \\
1 \\
1
\end{array}\right) \\
& \frac{d^{3} \hat{v}}{d \xi^{3}}\left(\begin{array}{c}
\tau^{2} \\
\alpha^{2} \tau^{2} \\
\alpha^{2} \tau^{2}
\end{array}\right)+\beta \frac{d f}{d \xi}\left(\begin{array}{c}
0 \\
0 \\
\alpha^{2} \tau^{4}
\end{array}\right)=0
\end{aligned}
$$

where the non-dimensional contact actions at the beam ends are defined as

$$
\hat{T}=\frac{T l^{2}}{E I}, \quad \hat{M}=\frac{M l}{E I} .
$$

The system of the non-dimensional field equation and boundary conditions (14), (15), (16) will be solved by a Galerkin approach in benchmark cases. The dependence of the non-dimensional natural angular frequencies on the quantities accounting for the physical properties of the beam will be discussed.

\section{Benchmark cases}

It is well known that Galerkin approach for the approximated solution of a differential problem needs a set of suitable comparison functions. The following benchmark cases will be investigated, for which known sets of comparison functions are at ease: a) a simply supported beam; b) a beam with two sliders, both orthogonal to the beam axis; c) a beam with a hinge at the left end and a slider orthogonal to the beam axis at the right end.

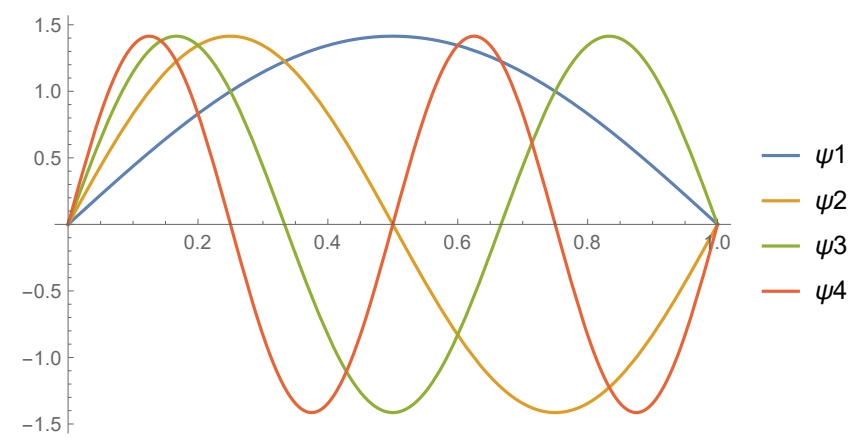

Figure 1: Set of comparison functions: sinus.

In the first case, it is known that the set of sinus functions in the domain $[0,1]$ is complete and provides well-working comparison functions. In addition, such functions verify also the non-standard 
kinematical boundary condition on the second derivative of the transverse displacement expressed by eq. $(15)_{3}$. The considered set, limited to four functions, is represented in fig. 1 and is given by

$$
\{\sin (\pi x), \sin (2 \pi x), \sin (3 \pi x), \sin (4 \pi x)\}
$$

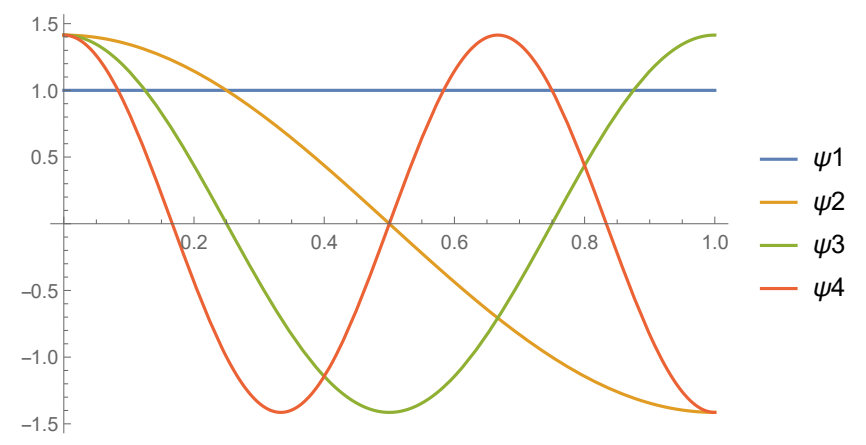

Figure 2: Set of comparison functions: cosinus.

In the second case, the set of cosinus functions in the domain $[0,1]$ will be adopted, which satisfies the non-standard mechanical boundary condition expressed by eq. (16) $)_{3}$. The considered set, limited to four functions, is expressed by

$$
\{1, \cos (\pi x), \cos (2 \pi x), \cos (3 \pi x)\}
$$

and is represented in fig. 2. Remark that the first function of the set, denoted $\psi 1$, is associated with the rigid body motion permitted to the beam.

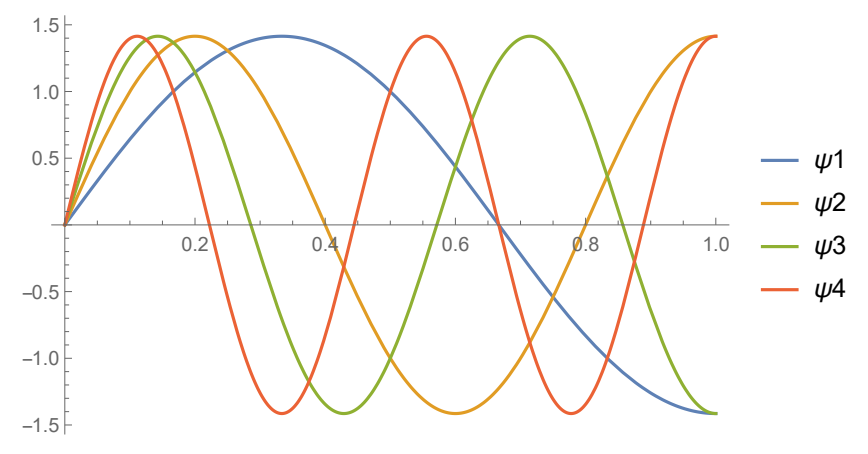

Figure 3: Set of comparison functions: rescaled sinus.

The third case is a combination of the first and the second, and a set of four suitable comparison functions on the domain $[0,1]$, represented in fig. 3 , is given by

$$
\left\{\sin \left(\frac{3 \pi x}{2}\right), \sin \left(\frac{5 \pi x}{2}\right), \sin \left(\frac{7 \pi x}{2}\right), \sin \left(\frac{9 \pi x}{2}\right)\right\}
$$

\subsection{Modulating linear dynamics}

The first investigation is about finding the first natural angular frequency (fundamental frequency) for the three benchmark cases, which are provided by the non-trivial solution of the homogeneous 
problems constituted by the system of eqs. (14), (15), (16), i.e., by assuming $q(x) \equiv a f(\xi) \equiv 0$. In the three cases, Galerkin approach by the help of four comparison functions, those in eqs. (18), (19), (20). Note that the comparison functions verify the boundary conditions, while they verify the field equation approximately; the approximated solution is attained by projecting the field equation onto the comparison functions, then solving the corresponding set of algebraic equations. When investigating natural vibration, the set of algebraic equations so obtained will have the form $\mathbf{M} \ddot{\mathbf{q}}+\mathbf{K q}=\mathbf{0}$, where $\mathbf{M}$ is called matrix of masses, $\mathbf{K}$ is called matrix of stiffnesses, and $\mathbf{q}$ is the vector of the amplitudes of the chosen comparison functions. In the cases investigated here, the non-dimensional axial force works as a modulating action on the fundamental frequency, in terms of the non-dimensional nonlocal Eringen parameter $\tau$ and of the participation factor between nonlocal and strain gradient elasticity $\alpha$. Thus, the stiffness matrix will be the sum of a purely elastic term and of a geometric term, as it is well known in the literature (e.g., Pignataro, Rizzi and Luongo [36]). By following the path of the fundamental frequency versus the modulating axial force, it is also possible to find the non-dimensional critical load inducing elastic static buckling. In the following, the mass and stiffness matrices for the three different boundary conditions that were investigated will be reported, and the relevant results for the fundamental frequency will be presented and shown in graphics.

\subsubsection{Hinged beam}

In this case, by the comparison functions of the set (18) provide the matrixes of masses and stiffnesses, as follows. According to the potential energy density $U_{1}$, it is

$$
\begin{aligned}
& M_{1}=\left(\begin{array}{cccc}
\frac{1}{2}\left(-\pi^{2} \gamma-1\right) & 0 & 0 & 0 \\
0 & \frac{1}{2}\left(-4 \pi^{2} \gamma-1\right) & 0 & 0 \\
0 & 0 & \frac{1}{2}\left(-9 \pi^{2} \gamma-1\right) & 0 \\
0 & 0 & 0 & \frac{1}{2}\left(-16 \pi^{2} \gamma-1\right)
\end{array}\right) \\
& K_{1}=\left(\begin{array}{cccc}
-\frac{1}{2} \pi^{2}\left(\pi^{4} \tau^{2}+\pi^{2}-\hat{N}\right) & 0 & 0 & 0 \\
0 & 2 \pi^{2}\left(\hat{N}-4\left(4 \pi^{4} \tau^{2}+\pi^{2}\right)\right) & 0 & 0 \\
0 & 0 & \frac{9}{2} \pi^{2}\left(\hat{N}-9\left(9 \pi^{4} \tau^{2}+\pi^{2}\right)\right) & 0 \\
0 & 0 & 0 & 8 \pi^{2}\left(\hat{N}-16\left(16 \pi^{4} \tau^{2}+\pi^{2}\right)\right)
\end{array}\right)
\end{aligned}
$$

while according to the potential energy density $U_{2}$ it is

$$
\begin{aligned}
& M_{2}=\left(\begin{array}{cccc}
\frac{1}{2}\left(-\pi^{2} \gamma-1\right) & 0 & 0 & 0 \\
0 & \frac{1}{2}\left(-4 \pi^{2} \gamma-1\right) & 0 & 0 \\
0 & 0 & \frac{1}{2}\left(-9 \pi^{2} \gamma-1\right) & 0 \\
0 & 0 & 0 & \frac{1}{2}\left(-16 \pi^{2} \gamma-1\right)
\end{array}\right) \\
& K_{2}=\left(\begin{array}{cccc}
-\frac{1}{2} \pi^{2}\left(\pi^{4} \alpha^{2} \tau^{2}+\pi^{2}-\hat{N}\right) & 0 & 0 & 0 \\
0 & 2 \pi^{2}\left(\hat{N}-4\left(4 \pi^{4} \alpha^{2} \tau^{2}+\pi^{2}\right)\right) & 0 & 0 \\
0 & 0 & \frac{9}{2} \pi^{2}\left(\hat{N}-9\left(9 \pi^{4} \alpha^{2} \tau^{2}+\pi^{2}\right)\right) & 0 \\
0 & 0 & 0 & 8 \pi^{2}\left(\hat{N}-16\left(16 \pi^{4} \alpha^{2} \tau^{2}+\pi^{2}\right)\right)
\end{array}\right)
\end{aligned}
$$

and according to the potential energy density $U_{3}$ it is

$$
\begin{aligned}
& M_{3}=\left(\begin{array}{cccc}
\frac{1}{2}\left(-\pi^{2} \gamma-1\right) & 0 & 0 & 0 \\
0 & \frac{1}{2}\left(-4 \pi^{2} \gamma-1\right) & 0 & 0 \\
0 & 0 & \frac{1}{2}\left(-9 \pi^{2} \gamma-1\right) & 0 \\
0 & 0 & 0 & \frac{1}{2}\left(-16 \pi^{2} \gamma-1\right)
\end{array}\right) \\
& K_{3}=\left(\begin{array}{cccc}
-\frac{1}{2} \pi^{2}\left(\pi^{4} \alpha^{2} \tau^{2}+\pi^{2}-\hat{N}\right) & 0 & 0 & 0 \\
0 & 2 \pi^{2}\left(\hat{N}-4\left(4 \pi^{4} \alpha^{2} \tau^{2}+\pi^{2}\right)\right) & 0 & 0 \\
0 & 0 & \frac{9}{2} \pi^{2}\left(\hat{N}-9\left(9 \pi^{4} \alpha^{2} \tau^{2}+\pi^{2}\right)\right) & 0 \\
0 & 0 & 0 & 8 \pi^{2}\left(\hat{N}-16\left(16 \pi^{4} \alpha^{2} \tau^{2}+\pi^{2}\right)\right)
\end{array}\right)
\end{aligned}
$$


It is apparent that the stiffness matrix associated with the potential energy density $\mathcal{U}_{1}$ does not contain the participation factor $\alpha$, hence it is not affected by strain gradient elasticity. On the other hand, the mass and stiffness matrices associated with the potential energy densities $\mathcal{U}_{2}, \mathcal{U}_{3}$ are the same, and would coincide with those in eq. (21) when $\alpha=1$.

By standard operations, the approximated non-dimensional fundamental angular frequencies are

$$
\Omega_{1}=\frac{\pi \sqrt{-\hat{N}+\pi^{4} \tau^{2}+\pi^{2}}}{\sqrt{\pi^{2} \gamma+1}}, \quad \Omega_{2}=\frac{\pi \sqrt{\pi^{4} \alpha^{2} \tau^{2}-\hat{N}+\pi^{2}}}{\sqrt{\pi^{2} \gamma+1}}, \quad \Omega_{3}=\frac{\pi \sqrt{\pi^{4} \alpha^{2} \tau^{2}-\hat{N}+\pi^{2}}}{\sqrt{\pi^{2} \gamma+1}}
$$

i.e., in closed form, where the subscript refers to the relevant potential energy density in eq. (4).

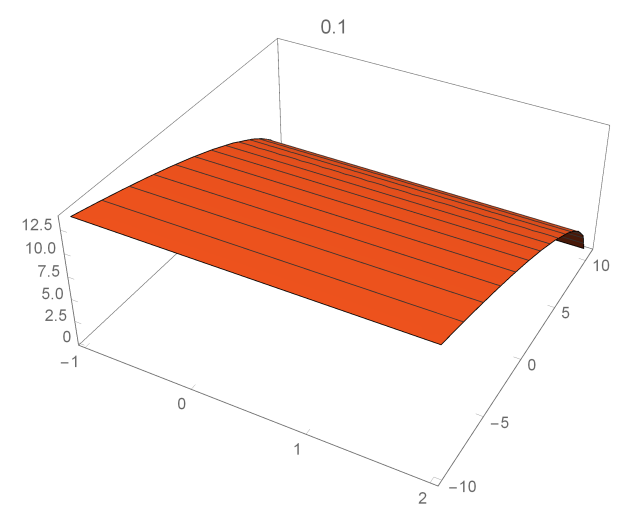

(a) $\tau=0.1$

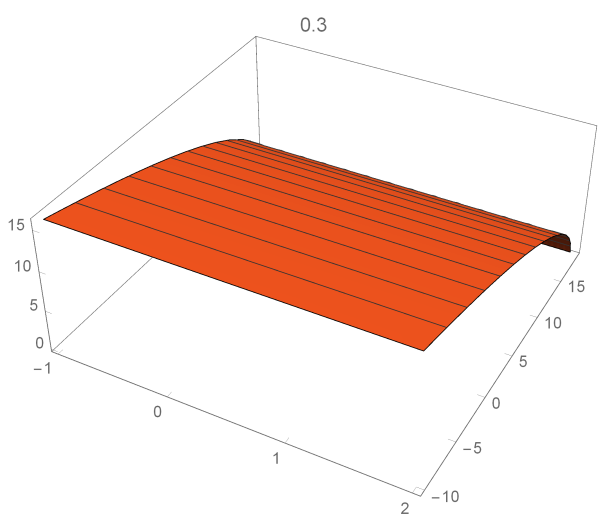

(c) $\tau=0.3$

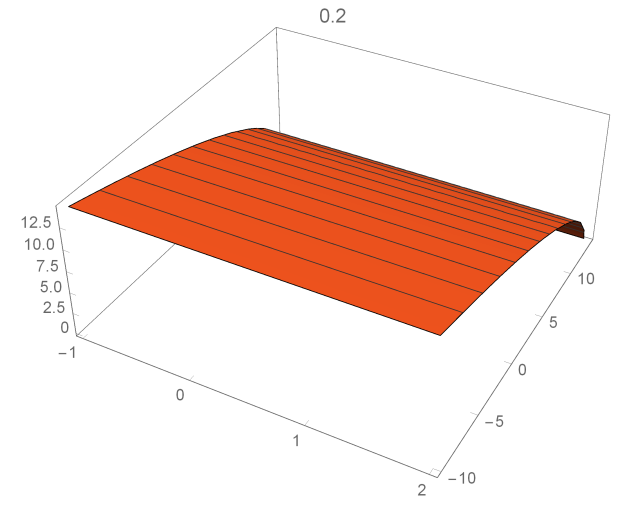

(b) $\tau=0.2$

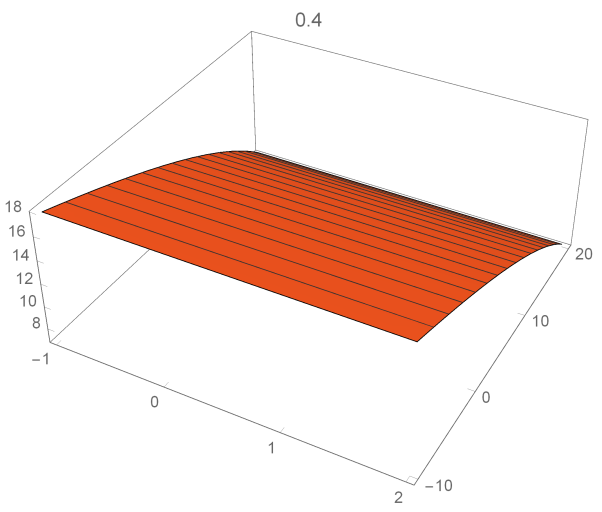

(d) $\tau=0.4$

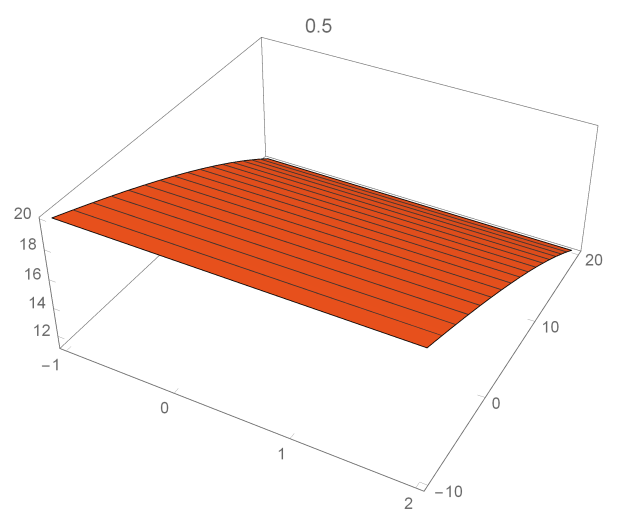

(e) $\tau=0.5$

Figure 4: Fundamental angular frequencies in terms of $\hat{N}, \alpha$, parametrised by $\tau$, for $\mathcal{U}_{1}$. 
In order to present some graphics for the fundamental frequencies provided by eq. (24), with no restrictions in generality it was set $\gamma=.01$. Indeed, the Euler-Bernoulli, purely flexible, model is descriptive enough of beam behaviour when the beams are slender enough, that is, if the characteristic dimension of their cross-section is much smaller than the length of its axis. If we admit, for the sake of simplicity, this ratio to be one tenth, the chosen value for $\gamma$, see eq. (13), appears to be reasonable enough. Then, it is possible to plot the variation of the non-dimensional fundamental frequencies $\Omega$ as a function of $\hat{N}$ and $\alpha$ as $\tau$ varies; the set of plots is shown in fig.4, pertaining to the potential energy density $\mathcal{U}_{1}$, and in fig. 5 and fig. 6 , pertaining to the potential energy densities $\mathcal{U}_{2}, \mathcal{U}_{3}$, respectively.

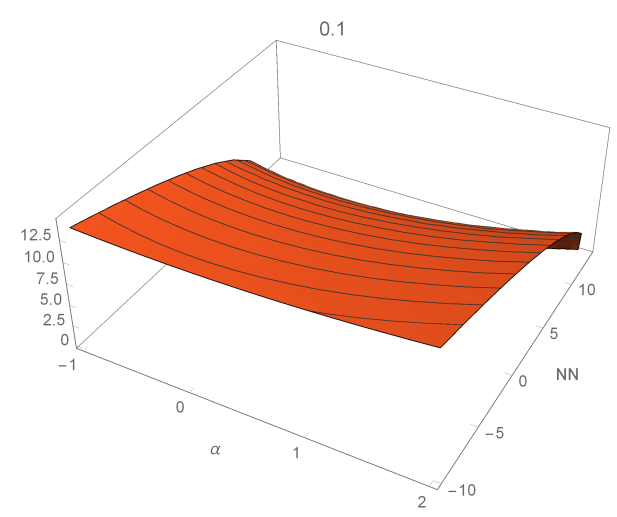

(a) $\tau=0.1$

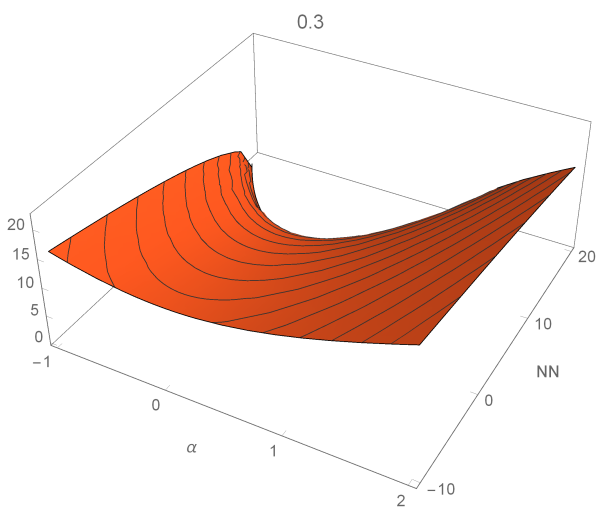

(c) $\tau=0.3$

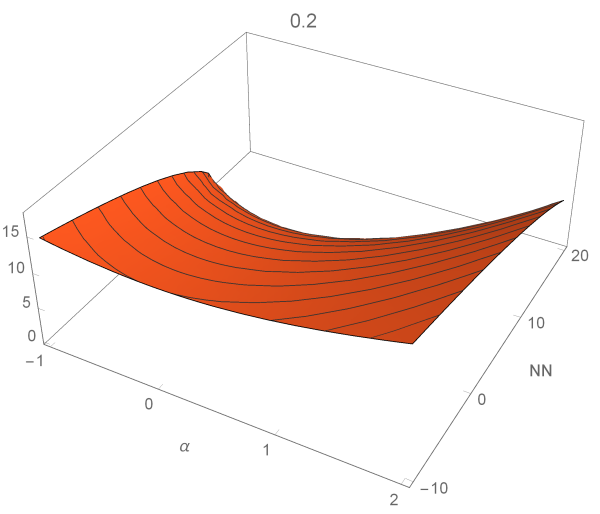

(b) $\tau=0.2$

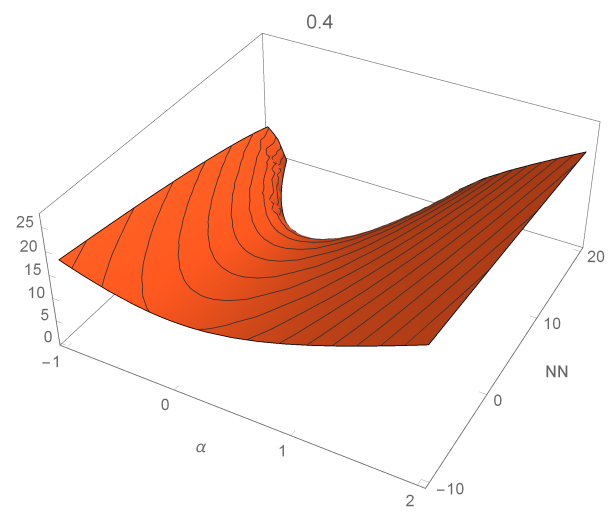

(d) $\tau=0.4$

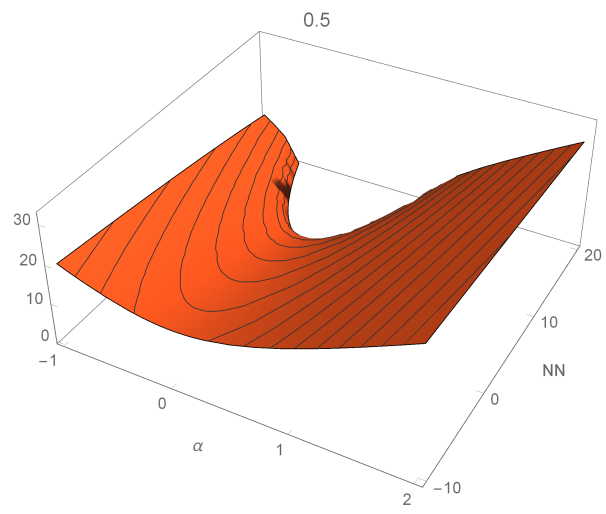

(e) $\tau=0.5$

Figure 5: Fundamental angular frequencies in terms of $\hat{N}, \alpha$, parametrised by $\tau$, for $\mathcal{U}_{2}$.

A look at the field equation (10) and to the closed-form expression of the approximated non- 
dimensional fundamental frequencies in eq. (24) shows that the results provided by the potential energy densities $\mathcal{U}_{2}, \mathcal{U}_{3}$ shall be the same, and this is confirmed by the graphs presented in figs. 5,6 .

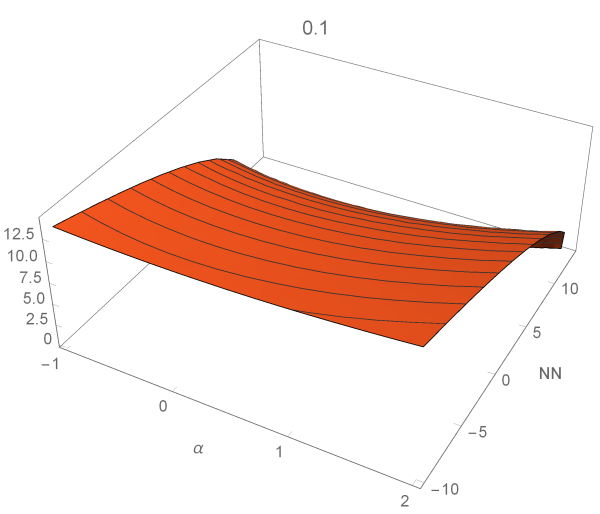

(a) $\tau=0.1$

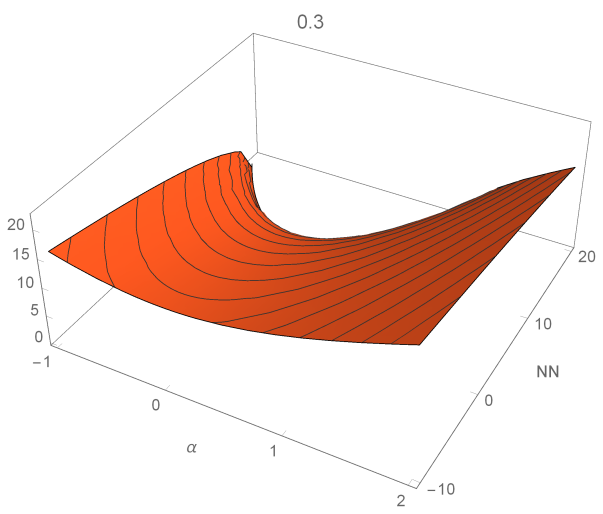

(c) $\tau=0.3$

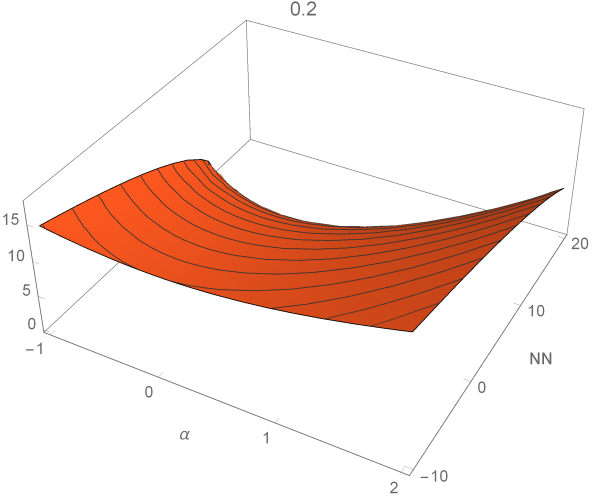

(b) $\tau=0.2$

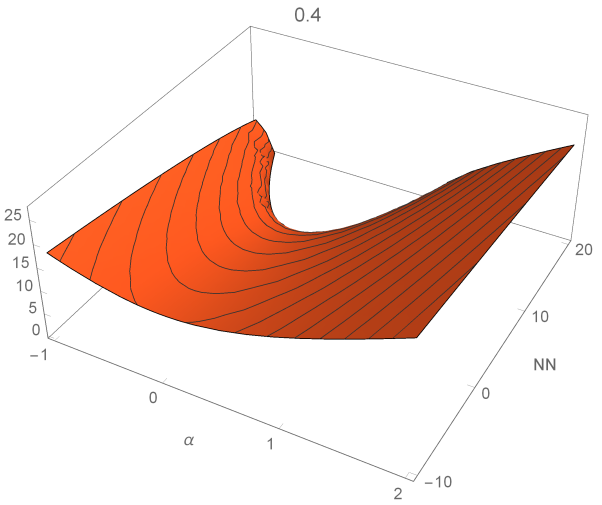

(d) $\tau=0.4$

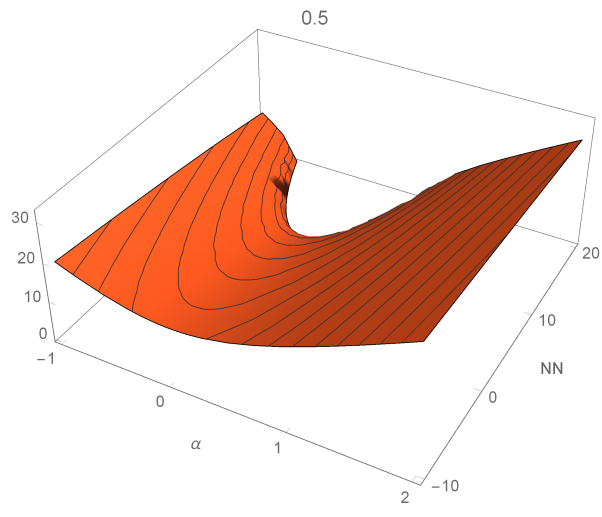

(e) $\tau=0.5$

Figure 6: Fundamental angular frequencies in terms of $\hat{N}, \alpha$, parametrised by $\tau$, for $\mathcal{U}_{3}$

Remark that, as already pointed out, the graphs in fig. 5 put into evidence that the fundamental frequency corresponding to the potential energy density $\mathcal{U}_{1}$ does not depend on the participation factor $\alpha$, and is monotonically decreasing with the modulating force (remember that $\hat{N}$ is positive in compression). This behaviour is maintained for increasing non-dimensional nonlocal elasticity parameter $\tau$, and the apparent effect is an increase in the fundamental frequency, i.e., a higher stiffness of the system. This is physically reasonable, since nonlocal effects, usually seen as softening, are activated 
by a transverse static (conservative) load, see the potential energy densities (4), while here no static transverse load is present, and the only transverse force is inertia. Roughly speaking, a greater value of the Eringen parameter implies the interaction of ever increasing long-range springs all on the same material point, thus a greater apparent material rigidity, and a greater fundamental frequency.

On the other hand, for the other potential energy densities, the fundamental frequency is in general non-monotonic with respect to both the participation factor $\alpha$ and the modulating force $\hat{N}$, while it is monotonically increasing with the nonlocal parameter $\tau$, for the same reason pointed out just above. The fundamental frequency decreases, then increases, passing from negative to positive values of the participation factor $\alpha$, and attains a minimum for $\alpha=0$, i.e., when no strain gradient effect is present. Indeed, the fundamental frequency depends on $\alpha^{2}$, see eq. (24), then its behaviour is symmetric with respect to $\alpha=0$. The fundamental frequency is quickly decreasing with the modulating force for small values of the nonlocal elasticity parameter, while this decrease is quite slow for high values of $\tau$, thus confirming once again that the material exhibits an apparent higher stiffness.

When $\alpha=1$, the three energy densities coalesce into a single one, and the variation of $\Omega$ with $\hat{N}$, parametrised by $\tau$, is shown in fig. 7: as the modulating force grows, the fundamental frequency decreases until it vanishes, as foreseen by the theory; the nonlocal parameter renders a stiffer behaviour.

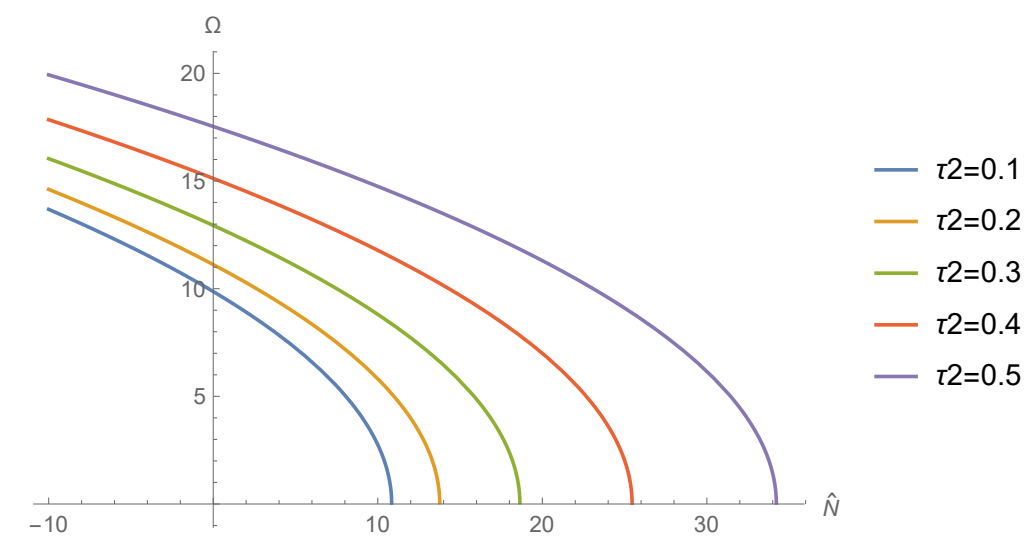

Figure 7: Variation of $\Omega$ with $\hat{N}$, with $\tau$ as a parameter.

The value of $\hat{N}$ inducing a null fundamental frequency is the buckling critical load; eq. (24) yields

$$
\hat{N}_{c 1}=\pi^{4} \tau^{2}+\pi^{2} \quad \hat{N}_{c 2}=\pi^{4} \alpha^{2} \tau^{2}+\pi^{2} \quad \hat{N}_{c 3}=\pi^{4} \alpha^{2} \tau^{2}+\pi^{2}
$$

and the variation of the critical loads with the material parameters of interest is shown in fig. 8. Once again, the results for the second and the third potential energy densities are undistinguishable, while those for $\mathcal{U}_{1}$ do not depend on the participation factor $\alpha$. The effect of nonlocal elasticity is stiffening, since here no static transverse load is present, and the only transverse force is inertia. 


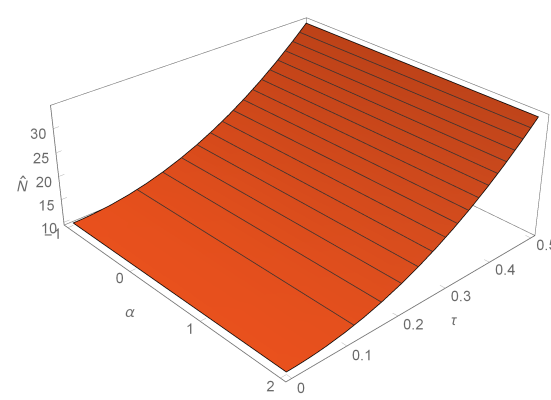

(a) $\mathcal{U}_{1}$

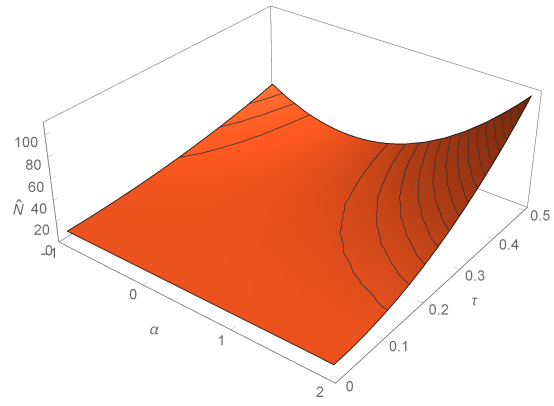

(b) $\mathcal{U}_{2}$

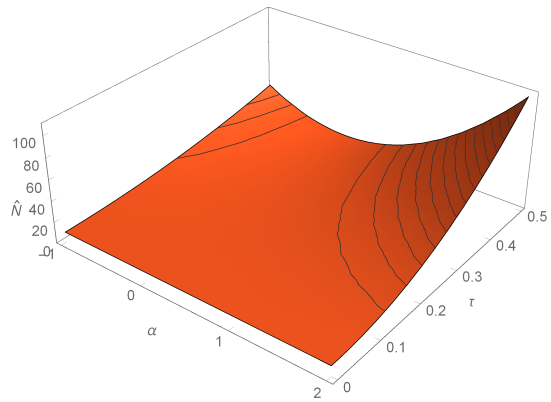

(c) $\mathcal{U}_{3}$

Figure 8: Critical load in terms of $\tau, \alpha$ for the three potential energy densities.

\subsubsection{A beam with two transverse sliders}

In this case, by the comparison functions of the set (19) provide the matrixes of masses and stiffnesses, as follows. According to the potential energy density $U_{1}$, it is

$$
\begin{aligned}
& M_{1}=\left(\begin{array}{cccc}
-1 & 0 & 0 & 0 \\
0 & -\pi^{2} \gamma-1 & 0 & 0 \\
0 & 0 & -4 \pi^{2} \gamma-1 & 0 \\
0 & 0 & 0 & -9 \pi^{2} \gamma-1
\end{array}\right) \\
& K_{1}=\left(\begin{array}{cccc}
0 & 0 & 0 & 0 \\
0 & -\pi^{2}\left(\pi^{4} \tau^{2}+\pi^{2}-\hat{N}\right) & 0 & 0 \\
0 & 0 & 4 \pi^{2}\left(\hat{N}-4\left(4 \pi^{4} \tau^{2}+\pi^{2}\right)\right) & 0 \\
0 & 0 & 0 & 9 \pi^{2}\left(\hat{N}-9\left(9 \pi^{4} \tau^{2}+\pi^{2}\right)\right)
\end{array}\right)
\end{aligned}
$$

while according to the potential energy density $U_{2}$ it is

$$
\begin{gathered}
M_{2}=\left(\begin{array}{cccc}
-1 & 0 & 0 & 0 \\
0 & -\pi^{2} \gamma-1 & 0 & 0 \\
0 & 0 & -4 \pi^{2} \gamma-1 & 0 \\
0 & 0 & 0 & -9 \pi^{2} \gamma-1
\end{array}\right) \\
K_{2}=\left(\begin{array}{cccc}
0 & 0 & 0 & 0 \\
0 & -\pi^{2}\left(\pi^{4} \alpha^{2} \tau^{2}+\pi^{2}-\hat{N}\right) & 0 \\
0 & 0 & 4 \pi^{2}\left(\hat{N}-4\left(4 \pi^{4} \alpha^{2} \tau^{2}+\pi^{2}\right)\right) & 0 \\
0 & 0 & 0 & 9 \pi^{2}\left(\hat{N}-9\left(9 \pi^{4} \alpha^{2} \tau^{2}+\pi^{2}\right)\right)
\end{array}\right)
\end{gathered}
$$

and according to the potential energy density $U_{3}$ it is

$$
\begin{gathered}
M_{3}=\left(\begin{array}{cccc}
-1 & 0 & 0 & 0 \\
0 & -\pi^{2} \gamma-1 & 0 & 0 \\
0 & 0 & -4 \pi^{2} \gamma-1 & 0 \\
0 & 0 & 0 & -9 \pi^{2} \gamma-1
\end{array}\right) \\
K_{3}=\left(\begin{array}{cccc}
0 & 0 & 0 & 0 \\
0 & -\pi^{2}\left(\pi^{4} \alpha^{2} \tau^{2}+\pi^{2}-\hat{N}\right) & 0 \\
0 & 0 & 4 \pi^{2}\left(\hat{N}-4\left(4 \pi^{4} \alpha^{2} \tau^{2}+\pi^{2}\right)\right) & 0 \\
0 & 0 & 0 & 9 \pi^{2}\left(\hat{N}-9\left(9 \pi^{4} \alpha^{2} \tau^{2}+\pi^{2}\right)\right)
\end{array}\right)
\end{gathered}
$$

to which the following expressions of the fundamental frequency correspond

$$
\Omega_{1}=\frac{\pi \sqrt{-\hat{N}+\pi^{4} \tau^{2}+\pi^{2}}}{\sqrt{\pi^{2} \gamma+1}}, \quad \Omega_{2}=\frac{\pi \sqrt{\pi^{4} \alpha^{2} \tau^{2}-\hat{N}+\pi^{2}}}{\sqrt{\pi^{2} \gamma+1}}, \quad \Omega_{3}=\frac{\pi \sqrt{\pi^{4} \alpha^{2} \tau^{2}-\hat{N}+\pi^{2}}}{\sqrt{\pi^{2} \gamma+1}}
$$

It is worth remarking that, even though we face a different benchmark scheme from the preceding one (doubly hinged beam), the expressions for the approximated non-dimensional fundamental frequencies, hence also of the critical loads, are the same. This is due to the fact that the comparison functions are simply shifted with respect to those for the doubly hinged beam, and the field equation, involving only 
even derivatives, acts in the same way on sinus and cosinus functions; Galerkin approach operates in the same way, hence it is reasonable to expect the same results.

\subsubsection{A beam with a transverse slider and a hinge}

This benchmark case may be seen as a combination of the preceding two. By the comparison functions of the set (20) provide the matrixes of masses and stiffnesses, as follows. According to the potential energy density $U_{1}$, it is

$$
\begin{aligned}
& M_{1}=\left(\begin{array}{cccc}
\frac{1}{8}\left(-9 \pi^{2} \gamma-4\right) & 0 & 0 & 0 \\
0 & \frac{1}{8}\left(-25 \pi^{2} \gamma-4\right) & 0 & 0 \\
0 & 0 & \frac{1}{8}\left(-49 \pi^{2} \gamma-4\right) & 0 \\
0 & 0 & 0 & \frac{1}{8}\left(-81 \pi^{2} \gamma-4\right)
\end{array}\right) \\
& K_{1}=\left(\begin{array}{cccc}
\frac{9}{128}\left(16 \hat{N} \pi^{2}-9 \pi^{4}\left(9 \pi^{2} \tau^{2}+4\right)\right) & 0 & 0 & 0 \\
0 & \frac{25}{128}\left(16 \hat{N} \pi^{2}-25 \pi^{4}\left(25 \pi^{2} \tau^{2}+4\right)\right) & 0 & 0 \\
0 & 0 & \frac{49}{128}\left(16 \hat{N} \pi^{2}-49 \pi^{4}\left(49 \pi^{2} \tau^{2}+4\right)\right) & 0 \\
0 & 0 & 0 & \frac{81}{128}\left(16 \hat{N} \pi^{2}-81 \pi^{4}\left(81 \pi^{2} \tau^{2}+4\right)\right)
\end{array}\right)
\end{aligned}
$$

while according to the potential energy density $U_{2}$ it is

$$
\begin{gathered}
M_{2}=\left(\begin{array}{cccc}
\frac{1}{8}\left(-9 \pi^{2} \gamma-4\right) & 0 & 0 & 0 \\
0 & \frac{1}{8}\left(-25 \pi^{2} \gamma-4\right) & 0 & 0 \\
0 & 0 & \frac{1}{8}\left(-49 \pi^{2} \gamma-4\right) & 0 \\
0 & 0 & 0 & \frac{1}{8}\left(-81 \pi^{2} \gamma-4\right)
\end{array}\right) \\
K_{2}=\left(\begin{array}{ccc}
\frac{9}{128}\left(16 \hat{N} \pi^{2}-9 \pi^{4}\left(9 \pi^{2} \alpha^{2} \tau^{2}+4\right)\right) \\
0
\end{array} \quad \frac{25}{128}\left(16 \hat{N} \pi^{2}-25 \pi^{4}\left(25 \pi^{2} \alpha^{2} \tau^{2}+4\right)\right)\right. \\
0 \\
0
\end{gathered}
$$

while according to the potential energy density $U_{3}$ it is

$$
\begin{aligned}
& M_{3}=\left(\begin{array}{cccc}
\frac{1}{8}\left(-9 \pi^{2} \gamma-4\right) & 0 & 0 & 0 \\
0 & \frac{1}{8}\left(-25 \pi^{2} \gamma-4\right) & 0 & 0 \\
0 & 0 & \frac{1}{8}\left(-49 \pi^{2} \gamma-4\right) & 0 \\
0 & 0 & 0 & \frac{1}{8}\left(-81 \pi^{2} \gamma-4\right)
\end{array}\right) \\
& K_{3}=\left(\begin{array}{cccc}
\frac{9}{128}\left(16 \hat{N} \pi^{2}-9 \pi^{4}\left(9 \pi^{2} \alpha^{2} \tau^{2}+4\right)\right) & 0 & 0 & 0 \\
0 & \frac{25}{128}\left(16 \hat{N} \pi^{2}-25 \pi^{4}\left(25 \pi^{2} \alpha^{2} \tau^{2}+4\right)\right) & 0 & 0 \\
0 & 0 & \frac{49}{128}\left(16 \hat{N} \pi^{2}-49 \pi^{4}\left(49 \pi^{2} \alpha^{2} \tau^{2}+4\right)\right) & \frac{81}{128}\left(16 \hat{N} \pi^{2}-81 \pi^{4}\left(81 \pi^{2} \alpha^{2} \tau^{2}+4\right)\right)
\end{array}\right)
\end{aligned}
$$

that provide the following expressions for the approximated non-dimensional fundamental frequencies

$$
\Omega_{1}=\frac{3 \pi \sqrt{81 \pi^{4} \tau^{2}+36 \pi^{2}-16 \hat{N}}}{4 \sqrt{9 \pi^{2} \gamma+4}}, \quad \Omega_{2}=\frac{3 \pi \sqrt{81 \pi^{4} \alpha^{2} \tau^{2}-16 \hat{N}+36 \pi^{2}}}{4 \sqrt{9 \pi^{2} \gamma+4}}, \quad \Omega_{3}=\Omega_{2}
$$

As it was made for the case of the doubly hinged beam, the values provided by eq. (33) are plotted as a function of $\hat{N}$ and $\alpha$, as $\tau$ varies, in fig. 9, fig. 10 and fig. 11 for the three potential energy densities, respectively. 


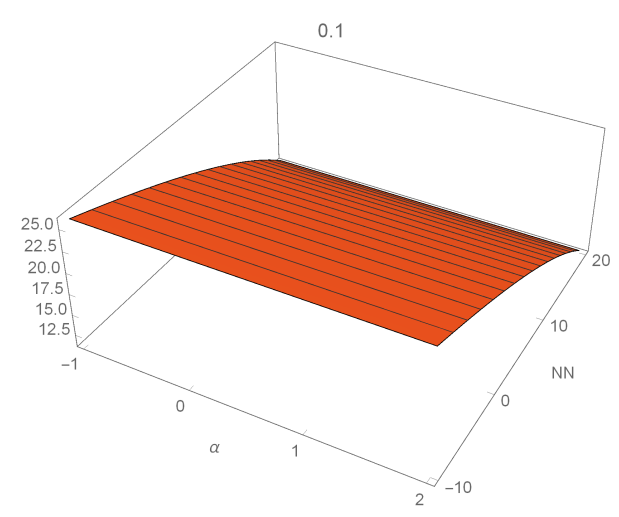

(a) $\tau=0.1$

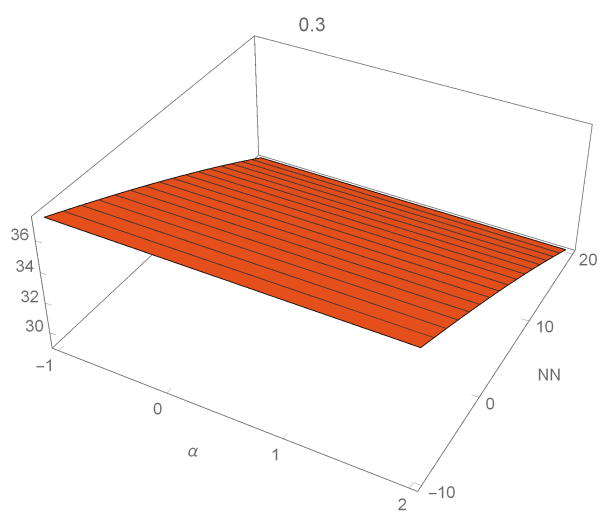

(c) $\tau=0.3$

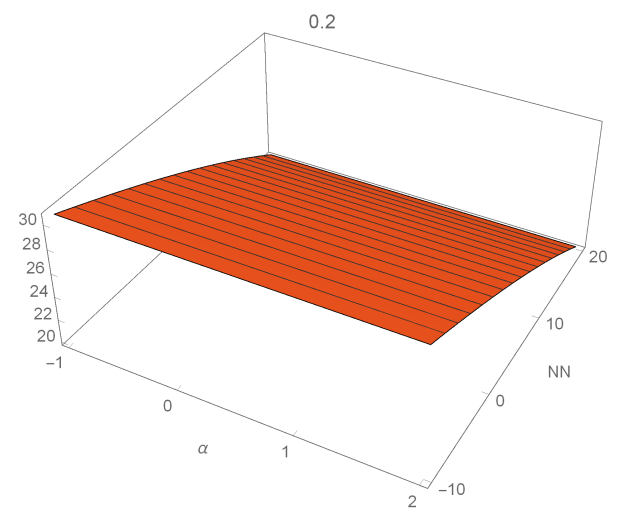

(b) $\tau=0.2$

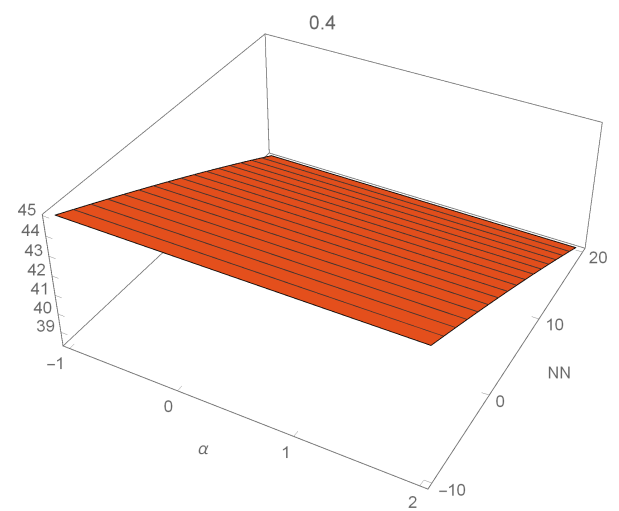

(d) $\tau=0.4$

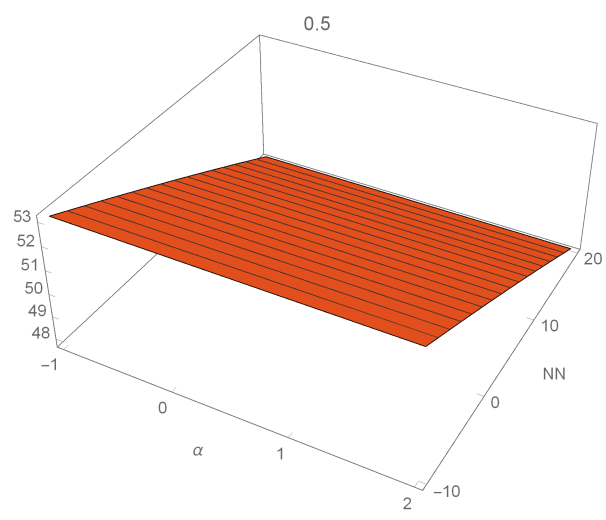

(e) $\tau=0.5$

Figure 9: Fundamental angular frequencies in terms of $\hat{N}, \alpha$, parametrised by $\tau$, for $\mathcal{U}_{1}$.

Remarks similar to those provided for the doubly hinged beam can be produced in this case as well, confirming that the model is robust, and that the obtained results are reliable. Remark that the fundamental frequency is always higher in this case with respect to that of the doubly hinged case, which was to be expected, since a slider is a stiffer constraint with respect to a hinge, and the global rigidity of the system is expected to be higher with respect to the simply supported beam. 


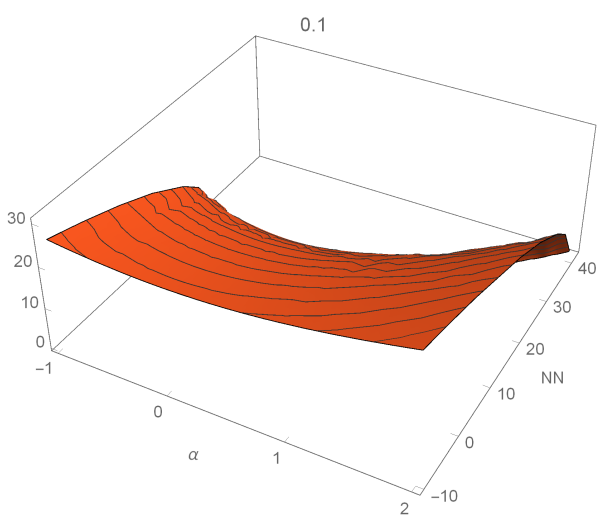

(a) $\tau=0.1$

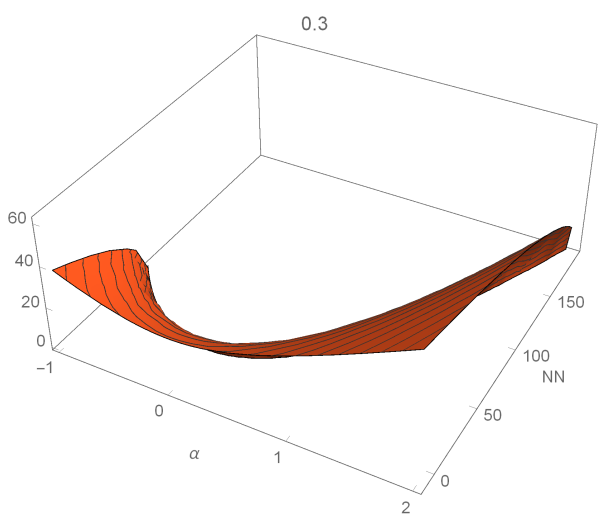

(c) $\tau=0.3$

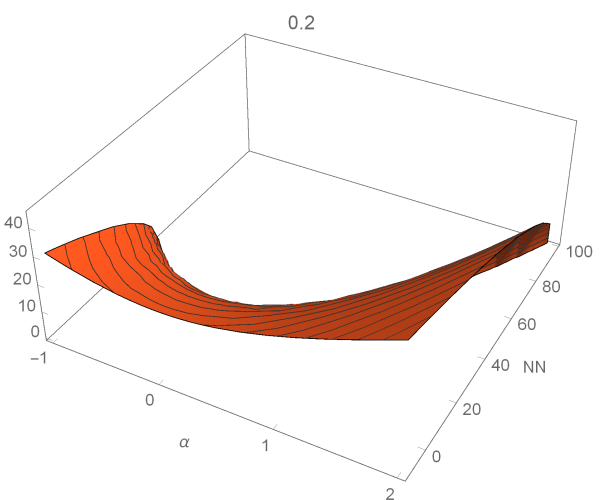

(b) $\tau=0.2$

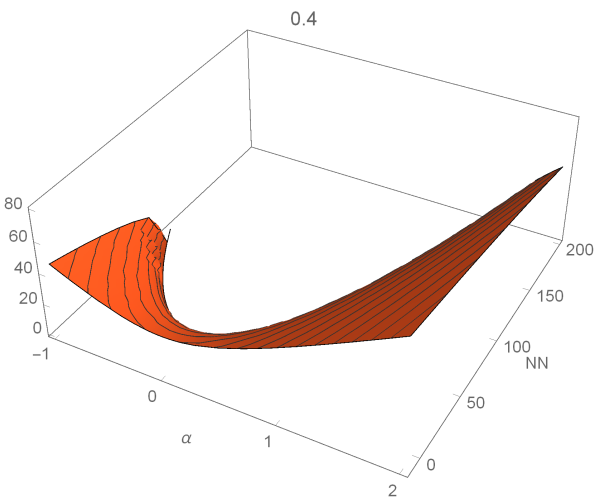

(d) $\tau=0.4$

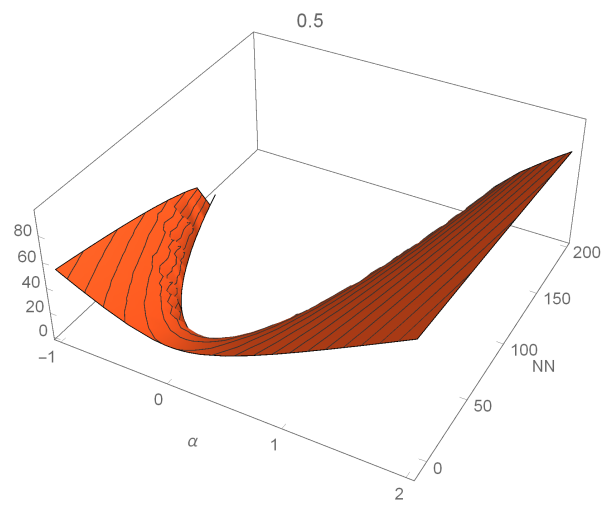

(e) $\tau=0.5$

Figure 10: Fundamental angular frequencies in terms of $\hat{N}, \alpha$, parametrised by $\tau$, for $\mathcal{U}_{2}$.

Also in this case, the results provided by $\mathcal{U}_{2}$ and $\mathcal{U}_{3}$ coincide, due to the shape of the potential energy density; again, remarks similar to those provided for the simply supported case can be brought, and the fundamental frequency is again higher; the values of the modulating forces are also rematkably greater than in the simply supported case. 


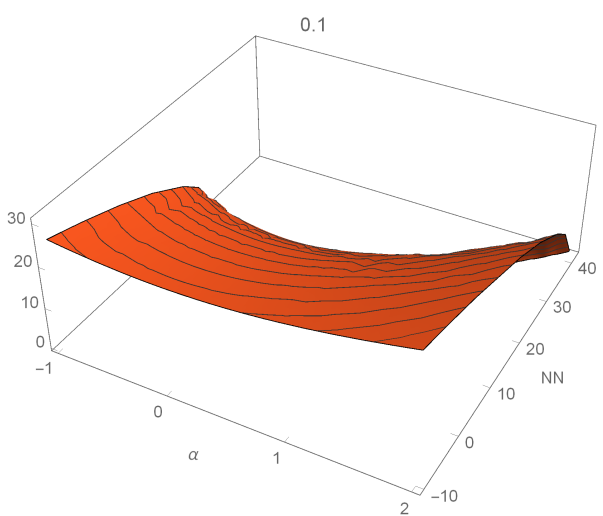

(a) $\tau=0.1$

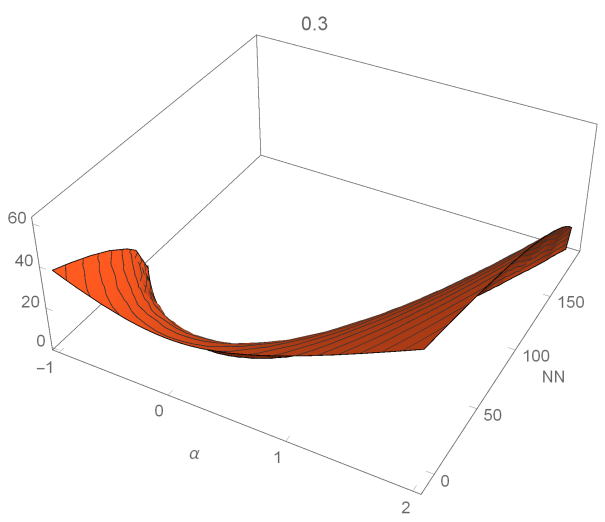

(c) $\tau=0.3$

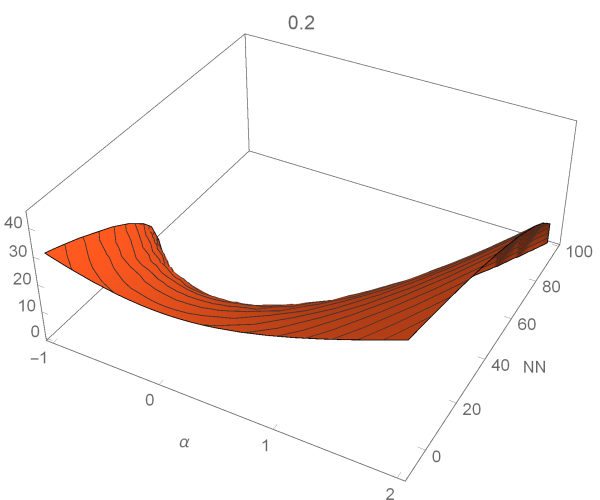

(b) $\tau=0.2$

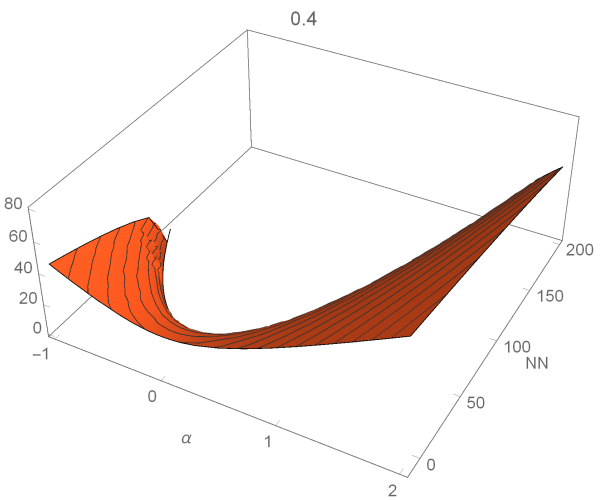

(d) $\tau=0.4$

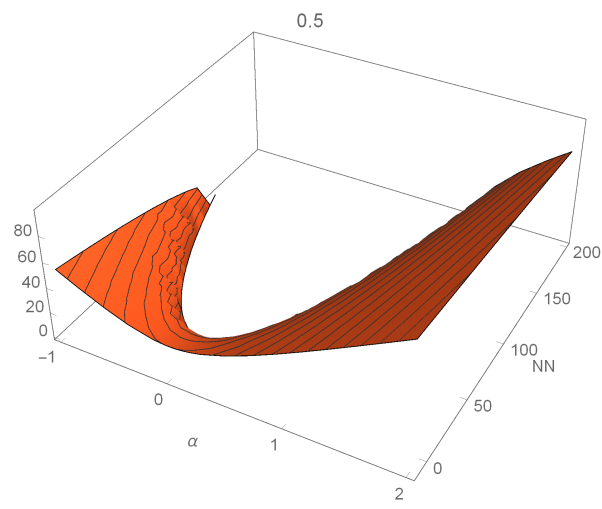

(e) $\tau=0.5$

Figure 11: Fundamental angular frequencies in terms of $\hat{N}, \alpha$, parametrised by $\tau$, for $\mathcal{U}_{3}$.

When $\alpha=1$, the three $\mathcal{U}_{i}$ coalesce into a single one, and the variation of $\Omega$ with $\hat{N}$, parametrised by $\tau$, is shown in fig. 12: as the modulating force grows, the fundamental frequency decreases until it vanishes, as foreseen by the theory; the nonlocal parameter renders a stiffer behaviour again. 


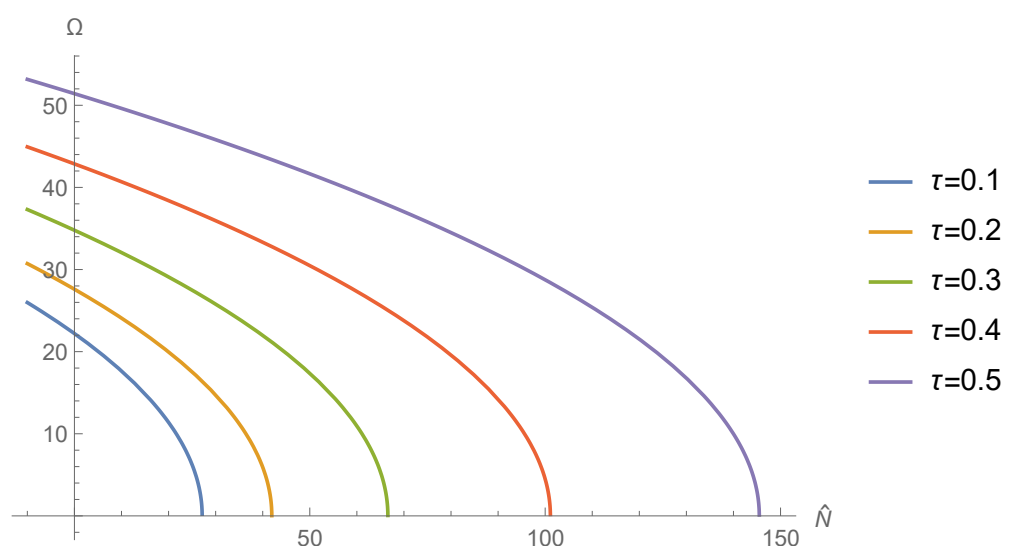

Figure 12: Variation of $\Omega$ with $\hat{N}$, with $\tau$ as a parameter.

The value of $\hat{N}$ inducing a null fundamental frequency is the buckling critical load; eq. (33) provides its values by making the denominator of the fundamental frequency vanish. The results are depicted in fig. 13 as $\alpha$ and $\tau$ vary.

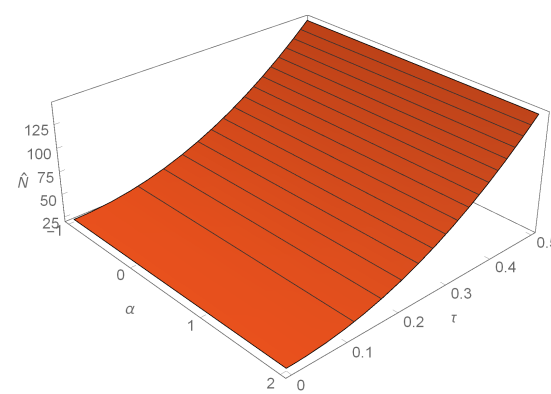

(a) $\mathcal{U}_{1}$

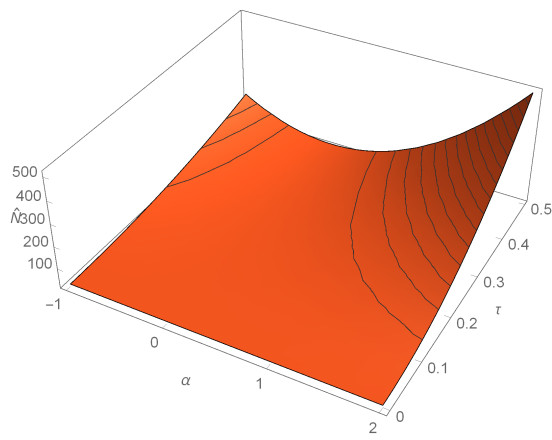

(b) $\mathcal{U}_{2}$

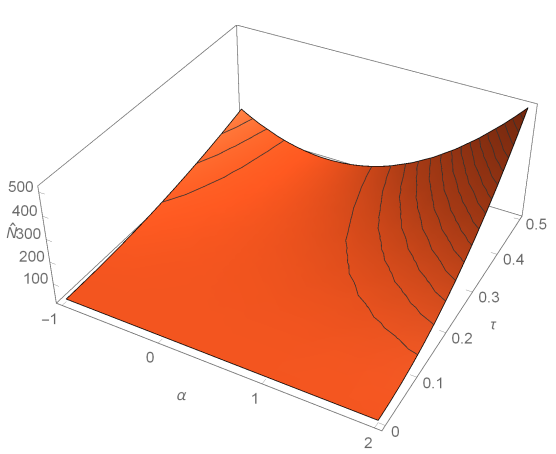

(c) $\mathcal{U}_{3}$

Figure 13: Critical load in terms of $\tau, \alpha$ for the three potential energy densities.

Similar to what was said on behalf of the fundamental frequencies, the critical load for the first potential energy density does not depend on the participation factor, and it grows as the nonlocal factor increase, for the reasons commented and thoroughly discussed above. The critical loads for the potential energy densities $\mathcal{U}_{2}, \mathcal{U}_{3}$ coincide, hence their graph is the same: the system grows stiffer when the nonlocal parameter increases, while it exhibits a non-monotonic behaviour with respect to the participation factor, attaining a minimum when $\alpha=0$, i.e., when no strain gradient effect is activated.

\section{$4 \quad$ Final remarks}

In this paper, we presented a first set of approximated results, obtained by means of a Galerkin approach, on the fundamental frequency and the critical loads of nanobeams for which a recent proposal of potential energy density combines the effects of nonlocal and strain gradient elasticity. By means of a set of few comparison functions, three benchmark cases were investigated, and the relevant results were presented and discussed. In this sense, these results are new, since they derive from an innovative proposal of combined constitutive relation. Thus, a comparison with existing literature is still under investigation, since the combination of nonlocality and strain gradient elasticity is still a debated topic. 
As a general result, there is a potential energy density for which the fundamental frequency and the critical load do not depend on the strain gradient elasticity participation factor; the other densities provide equal result, and the nonlocal effect has a stiffening effect on the fundamental frequencies and the critical loads, since the only transverse force is inertia.

Further developments of this investigation are in due course, and will consider the linear response to harmonic excitation, when the material properties vary, in a way similar to that examined here. The results will be reported elsewhere.

\section{References}

[1] Y.Q. Fu, H.J. Du, W.M. Huang, S. Zhang, M. Hu, TiNi-based thin films in MEMS applications: a review, Sensors and Actuators A: Physical 112:395-408 (2004)

[2] C. Ke, H.D. Espinosa, Numerical analysis of nanotube-based NEMS devices. Part I: electrostatic charge distribution on multiwalled nanotubes, Journal of Applied Mechanics 72:721-725 (2005)

[3] Z. Lee, C. Ophus, L.M. Fischer, N. Nelson-Fitzpatrick, K.L. Westra, S. Evoy, et al., Metallic NEMS components fabricated from nanocomposite Al-Mo films, Nanotechnology, 17(12):3063-3070 (2006)

[4] M. Li, H.X. Tang, M.L. Roukes, Ultra-sensitive NEMS-based cantilevers for sensing, scanned probe and very highfrequency applications, Nature Nanotechnology 2:114-120 (2007)

[5] J.N. Reddy, Nonlocal theories for bending, buckling and vibration of beams, International Journal of Engineering Science 45(2-8):288-307 (2007)

[6] H-T. Thai, A nonlocal beam theory for bending, buckling, and vibration of nanobeams, International Journal of Engineering Science 52:56-64 (2012)

[7] R. Barretta, F. Marotti de Sciarra, Analogies between nonlocal and local Bernoulli-Euler nanobeams, Archive of Applied Mechanics 85(1):89-99 (2015)

[8] R. Ansari, M Faraji Oskouie, R Gholami, F. Sadeghi, Thermo-electro-mechanical vibration of postbuckled piezoelectric Timoshenko nanobeams based on the nonlocal elasticity theory, Composites Part B 89:316-27 (2016)

[9] R. Barretta, L. Feo, R. Luciano, F. Marotti de Sciarra, An Eringen-like model for Timoshenko nanobeams, Composite Structures 139:104-110 (2016)

[10] R. Barretta, L. Feo, R. Luciano, F. Marotti de Sciarra, Application of an enhanced version of the Eringen differential model to nanotechnology, Composites Part B 96:274-280 (2016)

[11] Civalek Ö, Demir C, A simple mathematical model of microtubules surrounded by an elastic matrix by nonlocal finite element method, Applied Mathematics and Computation 289:335-352 (2016)

[12] B. Akgöz, Ö Civalek, Analysis of micro-sized beams for various boundary conditions based on the strain gradient elasticity theory, Archive of Applied Mechanics 82:423-443 (2012)

[13] R. Barretta, L. Feo, R. Luciano, F. Marotti de Sciarra, R. Penna, Functionally graded Timoshenko nanobeams: A novel nonlocal gradient formulation, Composites Part B 100:208-219 (2016)

[14] B. Akgöz, Ö Civalek, Longitudinal vibration analysis for microbars based on strain gradient theory, Journal of Vibration and Control 20: 606-616 (2014)

[15] B. Akgöz, Ö Civalek, Bending analysis of FG microbeams resting on Winkler elastic foundation via curvature gradient elasticity, Composite Structures 134:294-301 (2015) 
[16] B. Akgöz, Ö Civalek, Bending analysis of embedded carbon nanotubes resting on an elastic foundation using strain gradient theory, Acta Astronautica 119:1-12 (2016)

[17] M. Şimşek, Nonlinear free vibration of a functionally graded nanobeam using nonlocal curvature gradient theory and a novel Hamiltonian approach, International Journal of Engineering Science 105:12-27 (2016)

[18] B. Akgöz, Ö Civalek, Strain gradient elasticity and modified couple stress models for buckling analysis of axially loaded micro-scaled beams, International Journal of Engineering Science 49:1268-1280 (2011)

[19] Şimşek, M., Reddy, J.N. Bending and vibration of functionally graded microbeams using a new higher order beam theory and the modified couple stress theory International Journal of Engineering Science 64:37-53 (2013)

[20] M.A. Attia, F.F. Mahmoud, Modeling and analysis of nanobeams based on nonlocal-couple stress elasticity and surface energy theories, International Journal of Mechanical Sciences 105:126-34 (2016)

[21] K. Kiani, Thermo-elasto-dynamic analysis of axially functionally graded nonuniform nanobeams with surface energy, International Journal of Engineering Science 106:57-76 (2016)

[22] S. Gopalakrishnan, S. Narendar, Wave Propagation in Nanostructures, 59 NanoScience and Technology Springer International Publishing, Switzerland, 2013.

[23] Q. Wang, B. Arash, A review on applications of carbon nanotubes and graphenes as nano-resonator sensors, Computational Materials Science 82:350-360 (2014)

[24] R. Rafiee, R.M. Moghadam, On the modeling of carbon nanotubes: A critical review, Composites Part B 56:435-449 (2014)

[25] M.A. Eltaher, M.E. Khater, S.A. Emam, A review on nonlocal elastic models for bending, buckling, vibrations, and wave propagation of nanoscale beams, Applied Mathematical Modelling 40:4109-4128 (2016)

[26] K.F. Wang, B.L. Wang, T. Kitamura, A review on the application of modified continuum models in modeling and simulation of nanostructures, Acta Mechanica Sinica 32(1):83-100 (2016)

[27] R. Barretta, L. Feo, R. Luciano, F. Marotti de Sciarra, Variational formulations for functionally graded nonlocal Bernoulli-Euler nanobeams, Composite Structures 129:80-89 (2015)

[28] R. Barretta, M. Čanadija, F. Marotti de Sciarra, A higher-order Eringen model for Bernoulli-Euler nanobeams, Archive of Applied Mechanics 86:483-495 (2016)

[29] S. Woinowsky-Krieger, The effect of an axial force on the vibration of hinged bars, Journal of Applied Mechanics $17: 35-36(1950)$

[30] R.E.D. Bishop, W.G. Price, The vibration characteristics of a beam with an axial force, Journal of Sound and Vibration 59:237-244 (1974)

[31] A. Bokaian, Natural frequencies of beams under compressive axial loads, Journal of Sound and Vibration 126:49-65 (1988)

[32] A. Bokaian, Natural frequencies of beams under tensile axial loads, Journal of Sound and Vibration 142:481-498 (1990)

[33] N.G. Stephen, Beam compression under compressive axial load-upper and lower bound approximation, Journal of Sound and Vibration 131:345-350 (1989)

[34] Z.P. Bazant, L. Cedolin, Stability of structures, Oxford University Press, New York, 1991

[35] S.P. Timoshenko, J.M. Gere, Theory of elastic stability, McGraw-Hill, New York, 1961

[36] M. Pignataro, N. Rizzi, A. Luongo, Stability, Bifurcation and Postcritical Behaviour of Elastic Structures, Elsevier, Amsterdam, 1991 